(RESEARCH ARTICLE)

\title{
The effects of obesity along with other risk factors on type 2 diabetic patients in the northwest of Libya
}

\author{
Khaled Ali ALawaini $1{ }^{*}$ and Mustafa Ali Abugila ${ }^{2}$ \\ 1. Ph.D. student, Biochemistry department, Memorial University, Canada. \\ 2. Head, Biochemistry and Clinical Biochemistry department, University of Tripoli, Libya.
}

Publication history: Received on 04 November 2020; revised on 09 November 2020; accepted on 11 November 2020

Article DOI: https://doi.org/10.30574/wjarr.2020.8.2.0413

\begin{abstract}
Background

Type 2 diabetes has several causes, such as family history, age, physical inactivity, and unhealthy food. Obesity is a significant cause of type 2 diabetes (T2D). Microvascular and macrovascular complications are associated with longterm diabetes. However, the main objective in this study is to search for complications and risk factors related to diabetes.
\end{abstract}

Therefore, 472 diabetic patients with type 2 diabetes from northwest Libya participated in this study. Face-to-face interviews conducted using a questionnaire asking each patient information about name, age, sex, duration of disease, family history, and measured body mass index (BMI). A biochemical analysis (FPG, HbA1c, cholesterol, and triglyceride) was also performed on diabetic patients. Our results showed that $60.6 \%$ of type 2 diabetic patients had a positive family history. Poor glycemic control identified by high fasting plasma glucose (FPG) $186 \pm 9 \mathrm{mg} / \mathrm{dl}$, and glycated hemoglobin was (HbA1C) $8.36 \% \pm 1.8$.

Keywords: Obesity; Diabetes; Family history; Plasma glucose

\section{Introduction}

Diabetes Mellitus is a chronic disease (1) distinguished by high blood glucose concentration (hyperglycemia) (2). Diabetes, coined by Areaeus the Cappadocian, is a Greek word that means siphon, while Mellitus is a Latin word meaning sweet, was first named by the British surgeon John Rollo in 1798 (3). People with DM are more susceptible to different complications, as well as mortality (4). Likewise, several studies also showed that DM is a significant risk factor for heart disease and stroke (5). Undiagnosed or poorly controlled diabetes can result in lower limb amputation, blindness, and even kidney disease (6). In 2000, diabetes affected about 171 million people worldwide (7). In the United States, diabetes affected 22.3 million people, about $7 \%$ of the population in 2012. It was estimated that diabetes was responsible for $\$ 245$ billion in medical costs (8). In 2013, 382 million people were diagnosed with diabetes all over the World (9), and that number is expected to reach 552 million by 2030 (7). Additionally, worldwide, 10\% of the population will live with diabetes by 2030 (10). Type 1 diabetes mellitus is an absolute deficiency of insulin, leading to the destruction of beta cells, whereas type 2 diabetes mellitus, due to insulin resistance, leads to hyperglycemia (2). Insulin promotes the transportation of glucose through the membrane to the cells. However, in diabetic patients, Glu4 is usually reduced (11). Insulin resistance causes the release of free fatty acids (FFAs) from adipose tissue into the blood. As a result, the production and release of very-low-density lipoprotein (VLDL) in the liver cause dyslipidemia (12). Moreover, diabetes is commonly associated with metabolic disturbances in the production and clearance of plasma

\footnotetext{
${ }^{*}$ Corresponding author: Khaled Ali ALawaini

Ph.D. student, Biochemistry department, Memorial University, Canada.. 
lipoproteins. Furthermore, dyslipidemia may be a sign of diabetes. Diabetic dyslipidemia refers to low high-density lipoprotein (HDL), high triglycerides, and postprandial lipemia. However, dyslipidemia is mostly seen in patients with type 2 diabetes and cardiovascular disease (13). Type 2 accounts for $90-95 \%$ of diabetic patients (8). Most patients with type 2 are obese $(8,14)$. It has been reported that $80 \%$ of diabetic are obese $(15)$, and obesity is a significant risk factor for T2D (1). Additionally, family history, age (16), and lifestyle choices like a lack of physical activity and unhealthy food, are major risk factors of the disease (9). Besides type 1 and type 2 diabetes, gestational diabetes mellitus (GDM) is diabetes diagnosed in the second or third trimester of pregnancy. Moreover, other Specific types of diabetes result from many causes, such as neonatal diabetes or maturity-onset diabetes of the young (MODY), pancreatitis, cystic fibrosis, and chemical-induced diabetes after organ transplantations or in the treatment of infections (17). The other important consideration is prediabetic people. Those people are at high risk of developing type 2 and cardiovascular disease; it has been reported $70 \%$ of prediabetic people eventually become diabetic (10). Prediabetes means blood glucose concentration is higher than normal but lower than those diagnosed with diabetes, defined as (OGTT: 140-199 $\mathrm{mg} / \mathrm{dL}$ ) or (IFG 100-125 mg/dL). However, this intermediate can increase the risk of diabetes and associated CV complications. Therefore, early diagnosis and treatment of IGT and IFG are necessary to prevent diabetes and its associated morbidity and mortality (18). Patients diagnosed with diabetes based on fasting plasma glucose (FPG) levels or based on glucose tolerance test (2-h GTT) levels during a 75-g oral, or by glycosylated hemoglobin (HbA1c) criteria, which reflect long term glucose concentration for 2 to 3 months (18). Diabetes is a significant disease in Libya. As a result, uncontrolled DM can lead to several dangerous complications such as retinopathy, nephropathy, neuropathy, and cardiovascular diseases (19). Consequently, the purpose of this study search for diabetes and associated complications that can lead to morbidity and mortality and reduce disease incidence.

\section{Methods}

A total of 472 diabetic patients with type 2 diabetes from northwest Libya participated in this study. The study was conducted from November 2008 to April 2009. However, face-to-face interviews were conducted using a questioner asking each patient information about name, age, sex, duration of disease, family history, and measured height and body weight using a height scale. Body mass index (BMI) was calculated according to the World health organization recommended normal (18.5-24.9 kg/m²), overweight (25-29.9 kg/m²), obesity more than $\left(30 \mathrm{~kg} / \mathrm{m}^{2}\right)$. As a result, weight in $\mathrm{kg}$ was divided by height in meter squared $\left(\mathrm{kg} / \mathrm{m}^{2}\right)$. Additionally, after overnight fasting, $5 \mathrm{ml}$ of venous blood was collected from each patient for biochemical analysis such as blood glucose, urea, creatinine, cholesterol, triglyceride, and HbA1c. However, urine samples were also collected and checked for the presence of albumin and ketone bodies.

\subsection{Chemicals used in these experiments}

Urea, creatinine, cholesterol, and triglyceride were measured by spectrophotometer (Clima plus-825-Spain) with the use of Fluitest-Bicon diagnostic Heck 8 kits. In contrast, HbA1c was measured by Cobas Integra (400 Plus- Switzerland) with Roche diagnostic $\mathrm{GmbH}$ )., and urine strips (Combi-screen- Test strips Germany) were used for the analysis of urine samples.

\subsection{Statistical analysis}

Analysis of data was performed with computer software (SPSS. Version 16.0, and excel 2007). ANOVA test was done to test the relationship between different biochemical tests. The (Chi) test was performed to test the statistical significance of the difference in various parameters.

\section{Results}

A sample of 472 patients diagnosed with type 2 diabetes participated in this study. The mean age group was $56.55 \pm 11.8$ years old. However, the average duration of the disease was 7.64 years.

Table 1 shows the distribution of age grouping of the sample population in T2DM.

\begin{tabular}{|l|l|l|}
\hline Age groups & Number of patients & Percentage \% \\
\hline $30-39$ & 27 & 5.8 \\
\hline $40-49$ & 116 & 24.5 \\
\hline $50-59$ & 139 & 29.5 \\
\hline$>60$ & 190 & 40.2 \\
\hline
\end{tabular}




\begin{tabular}{|l|l|l|}
\hline Total & 472 & $100 \%$ \\
\hline
\end{tabular}

Table 1 shows the distribution of diabetic patients according to age groups. However, the age group between 30-39 years had the lowest number of patients. Thus could confirm that type 2 usually come after 40 years old. However, the number of patients increased with age.

Table 2 percentage of T2 in females and males of the studied samples.

\begin{tabular}{|l|l|l|}
\hline Sex & Number of patients & Percentage (\%) \\
\hline Female & 270 & 57.2 \\
\hline Male & 202 & 42.8 \\
\hline Total & 472 & $100 \%$ \\
\hline
\end{tabular}

The proportion of female patients was observed to be $42.8 \%$ males to $57.2 \%$ females with a ratio male to female $0.748: 1$.

Table 3 the mean and standard deviation (STD) of the biochemical parameter.

\begin{tabular}{|l|l|l|l|l|l|}
\hline & BMI $\left(\mathbf{k g} / \mathbf{m}^{2}\right)$ & Mean FBS & Mean HbA1C & Mean cholesterol(mg/dl) & Mean TG (mg/dl) \\
\hline Mean & 29.84 & 186 & 8.36 & 192.2 & 163.5 \\
\hline STD & 4.8 & 69 & 1.8 & 49.1 & 82.3 \\
\hline Total & 472 & 472 & 228 & 246 & 246 \\
\hline
\end{tabular}

Biochemical parameters of 472 diabetic patients with type 2 showed the average of blood glucose $186 \mathrm{mg} / \mathrm{dl}$, cholesterol $192.2 \mathrm{mg} / \mathrm{dl}$, triglyceride $163.5 \mathrm{mg} / \mathrm{dl}, \mathrm{HbA} 1 \mathrm{c} 8.36 \%$, and BMI $29.84 \mathrm{~kg} / \mathrm{m}^{2}$.

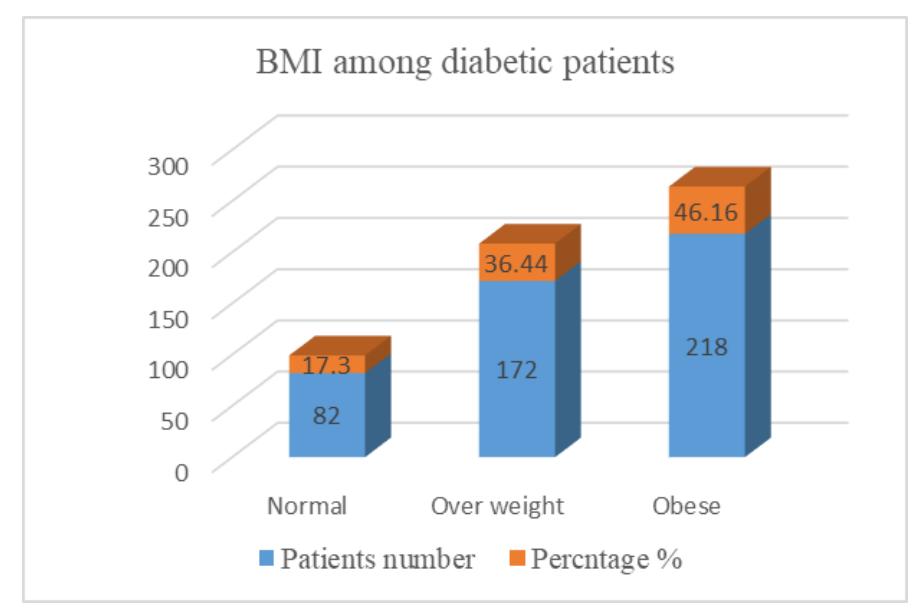

Figure 1 classification of diabetic patients according to BMI

Out of 472 diabetic patients, most 390 (82.6\%) were either overweight or obese. Moreover, $36.4 \%$, $46.2 \%$ overweight, and obese. However, only $1.1 \%$ and $16.3 \%$ were underweight and normal weight, respectively. Females were more predominant than males in this study $(58.2 \%, 42.8 \%)$, respectively. Statistically, there was a significant difference between normal weight compared to overweight and obese $(\mathrm{P}<0.05)$.

Table 4 percentage of cholesterol and triglyceride in diabetic patients.

\begin{tabular}{|l|l|l|l|l|}
\hline \multicolumn{5}{|c|}{ Cholesterol } \\
\hline Number of samples & Normal & \% & Abnormal & $\%$ \\
\hline 246 & 146 & 59.3 & 100 & 40.7 \\
\hline \multicolumn{5}{|c|}{ Triglyceride } \\
\hline Number of samples & Normal & $\%$ & Abnormal & $\%$ \\
\hline 246 & 175 & 71.1 & 71 & 28.9 \\
\hline
\end{tabular}


Table 5 means of cholesterol level in the different age group.

\begin{tabular}{|l|l|l|l|}
\hline Age group (Years) & Mean of Cholesterol & Range (mg/dl) & STD \\
\hline $20-29$ & 152.5 & $111-194$ & $58 . .6$ \\
\hline $30-39$ & 168.12 & $118-246$ & 33.6 \\
\hline $40-49$ & 198.9 & $90-464$ & 53.4 \\
\hline $59-59$ & 190.1 & $90-311$ & 47.8 \\
\hline$>60$ & 194.7 & $88-370$ & 49.0 \\
\hline
\end{tabular}

Statistically, not significant $(\mathrm{P}>0.05)$. Between the mean of blood cholesterol levels in different age groups.

Table 6 means of triglyceride in the different age group.

\begin{tabular}{|l|l|l|l|}
\hline Age group (Years) & Mean of TG & Range mg/dl & STD \\
\hline $20-29$ & 124 & $115-133$ & 12.7 \\
\hline $30-39$ & 163.19 & $94-239$ & 47.2 \\
\hline $40-49$ & 173 & $58-820$ & 111.5 \\
\hline $59-59$ & 185.8 & $60-429$ & 90.9 \\
\hline$>60$ & 144 & $53-333$ & 56.5 \\
\hline
\end{tabular}

There are significant differences in serum triglyceride levels between the mean serum triglyceride levels in different age groups $(\mathrm{P}<0.05)$

Table 7 distribution of type 2 diabetes on the type of treatment.

\begin{tabular}{|l|l|l|l|l|l|l|}
\hline Disease period & \multicolumn{2}{l}{ Number of patients } \\
\hline (Years) & No medication & $\mathbf{\%}$ & Oral medication & $\mathbf{\%}$ & Insulin & $\mathbf{\%}$ \\
\hline$<1$ & 36 & 47.4 & 36 & 47.4 & 4 & 5.2 \\
\hline $1-3$ years & 12 & 12.1 & 64 & 64.6 & 23 & 23.2 \\
\hline 4-8 years & 6 & 4.7 & 82 & 63.6 & 41 & 31.8 \\
\hline 9-13 years & 1 & 1.3 & 27 & 36 & 47 & 62.7 \\
\hline 14-19 years & 0 & 0 & 15 & 31.3 & 33 & 68.7 \\
\hline 20-24 years & 0 & 0 & 6 & 19.4 & 25 & 80.6 \\
\hline$>25$ years & 0 & 0 & 2 & 14.3 & 12 & 85.7 \\
\hline Total & $\mathbf{5 5}$ & $\mathbf{1 1 . 7}$ & $\mathbf{2 3 2}$ & $\mathbf{4 9}$ & $\mathbf{1 8 5}$ & $\mathbf{3 9 . 3}$ \\
\hline
\end{tabular}

Most diabetic patients were taking oral medication (49\%), whereas $11.7 \%$ without any prescription. Unexpected results showed that $39.3 \%$ of patients changed their treatment to insulin, as demonstrated in table 7.

Table 8 different parameters in the studied samples.

\begin{tabular}{|l|l|l|l|l|l|}
\hline Parameter & Normal & $\mathbf{\%}$ & Abnormal & \% & Total \\
\hline HbA1c & 50 & 22.7 & 170 & 77.3 & 220 \\
\hline Urea & 248 & 96.9 & 8 & 3.1 & 256 \\
\hline Creatinine & 249 & 97.3 & 7 & 2.7 & 256 \\
\hline Urine ketone & 113 & 92.7 & 9 & 7.3 & 122 \\
\hline Urine Albumin & 119 & 97.5 & 3 & 2.5 & 122 \\
\hline
\end{tabular}

Biochemical parameters revealed that $77.3 \%$ of patients had poor glycemic control, HbA1c more than $7 \%$. With time, this can lead to more complications. Furthermore, poor glycemic control could reflect high creatinine levels, uremia, and albuminuria $(2.7 \%, 3.1 \%$, and $2.5 \%)$ in diabetic patients in this study. 


\section{Discussion}

According to the WHO, the prevalence of T2D in Tunisia was 15.1\%. In Algeria, the diabetes prevalence was 8.2\%, 16.1\% in Oman, and $16.7 \%$ in Qatar. However, the highest rates of T2D were reported in Bahrain, 25.7\%, Saudi Arabia 23.7\%, and the United Arab Emirates, 17.1\%. In Spain and Switzerland, it was $13.2 \%$ and 11.5\%, respectively (20). This study showed the prevalence of T2D was higher in females than males $57.2 \%$ vs. $42.8 \%$, and the lowest prevalence of type 2 was seen in the age group between $30-39$ old, only in 27 individuals, 5.8\% of the total age grouping population. Therefore, this could confirm that type 2 usually occurs after 40 years old. In this study, the prevalence of type 2 increased with age group and reached peaked after 60 years old. As a result, this could explain that age is a significant risk factor for $\mathrm{T} 2$ diabetes.

Another important finding from this study is the poor glycemic control as described by the mean of plasma glucose and glycated hemoglobin (mean FPG $186 \pm 9 \mathrm{mg} / \mathrm{dl}$, HbA1C 8.36\% \pm 1.8 ). Another study was also carried out in Tripoli in 2013; out of 523, only $21.8 \%$ of patients were on glycemic control (A1C $<7$ ), whereas poor glycemic control was seen in 78.2\% with mean HbA1c 8.9\%. (21). A similar study was carried out in Tripoli, Libya, on 551 diabetic patients; $68.2 \%$ of diabetic patients showed poor glycemic control $(\mathrm{A} 1 \mathrm{C} \geqslant 7$ ), whereas the remaining $31.8 \%$ were on glycemic control (A1C <7) (22). Our study showed a considerable variation in BMI for 472 patients with a mean BMI of $29.84 \mathrm{~kg} / \mathrm{m}^{2}$; normal weight was observed in $17.3 \%$ of patients (less than $24.9 \mathrm{~kg} / \mathrm{m}^{2}$ ), overweight was in $36.44 \%$, while the remaining $46.16 \%$ were obese. In general, (82.6\%) were either overweight or obese; the remaining 16.3\% were normal. As a result, there was a significant difference between normal weight, overweight, and obesity $(\mathrm{P}<0.05)$, which explains high triglyceride, cholesterol and reflecting poor glycemic control. Moreover, obesity was more predominant in females than males in this study, $58.2 \%, 42.8 \%$. In Tripoli, El-Shareif (23) also found that out of the 370 patients had a mean BMI of $30.8 \pm 8.4$, measured as $\mathrm{kg} / \mathrm{m}^{2}, 20.5 \%$ had a BMI of normal body weight. However, overweight and obese had been seen in 31.9\% and $47.6 \%$, respectively. Another study was carried out in Misurata, $200 \mathrm{~km}$ from Tripoli to the East, which found that obesity was more prevalent in $56.8 \%$ of type 2 and was more pronounced among females than males (19). In the recent study, cholesterol and triglyceride levels were performed for 246 (52.11\%) patients, and mean cholesterol level and triglyceride (TG) were $192.2 \pm 49.1 \mathrm{mg} / \mathrm{dl} 163.5 \pm 82.3 \mathrm{mg} / \mathrm{dl}$, respectively. Our finding revealed that the mean of cholesterol, as well as TG, increased significantly with age. There was a significant difference between the mean serum triglyceride levels in different age groups $(\mathrm{P}<0.05)$, which explains the higher level of obesity and diabetes. Diabetes is commonly associated with dyslipidemia, including low high-density lipoprotein (HDL) and increased triglycerides leading to a disturbance of lipid metabolism. However, this could be due to insulin resistance and hyperglycemia (13). As a result, there is an increased risk of vascular disease, which can lead to death. A similar study reported high cholesterol and TG levels, $236+3.16 \mathrm{vs} .248+2.8 \mathrm{mg} / \mathrm{dl}$, respectively, when 474 diabetic patients were tested for lipid profile compared to our research. Our data demonstrated that the patients' period started from less than a year up to more than 20 years. Our finding was that 185 (39.3\%) were treated with insulin,.In contrast, 232 (49\%) were taken oral hypoglycemic medications, and the remaining $55(11.7 \%)$ were treated with no cure. However, most $76(16.1 \%)$ patients were diagnosed recently (less than a year); furthermore, most patients, $64.4 \%$ of total diabetic patients, were located in the age group between 1 to 8 years, in which $14.4 \%$ were started to use insulin; which explained poor glycemic control. However, this poor glycemic control could lead to more complications. A study similar to this in Libya also found that $32.6 \%$ of patients used insulin and $58 \%$ oral hypoglycemic treatment. However, this poor control over time leads to microvascular or macrovascular complications. As a result, a total of 122 urine samples were tested for ketone and albumin. We found that $8.1 \%$ had ketoacidosis and $2.2 \%$ with albuminuria. Along with 48 patients, $9.8 \%$ of all patients included a combination of retinopathy $(33.3 \%)$ and $(20.8 \%)$ cataract, neuropathy $(35.5 \%)$, nephropathy (1.5\%), and 8.3\% with cardiovascular disease. Another significant finding in the recent study revealed that $60.6 \%$ of patients with $\mathrm{T} 2 \mathrm{had}$ a positive family history and confirmed that family history is a considerable risk factor in T2D. However, only parents were considered; either father or mother had diabetes.

\section{Conclusion}

The prevalence of obesity, family history, and age as risk factors in type 2 diabetes is very high. Poor glycemic control (Mean of FPS $186 \mathrm{mg} / \mathrm{dl}$, HbA1c 8.36\%) is associated with hyperlipidemia. Knowledge and lifestyle factors play an essential role in patients' decisions regarding the prevention and treatment of diabetes and its complications.

\section{Compliance with ethical standards}

\section{Acknowledgments}

We are grateful to all people who participated in this study and all diabetic centers for their help and corporation. 


\section{Disclosure of conflict of interest}

There is no conflict of interest.

\section{Statement of informed consent}

All patients in this study gave their informed consent.

\section{References}

[1] Malone J, Hansen B. Does obesity cause type 2 diabetes mellitus (T2DM)? Or is it the opposite? Pediatric Diabetes. 2019; 20: 5-9.

[2] Schmidt AM. Highlighting diabetes mellitus, the epidemic continues. Arteriosclerosis, thrombosis, and vascular biology. 2018; 38(1): 1-8.

[3] Lakhtakia R. The history of diabetes mellitus. Sultan Qaboos univ med journal.2013; 13(3): 368-370.

[4] Chuah LL, Papamargaritis D, Pillai D, Krishnamoorthy A, le Roux C. Morbidity and mortality of diabetes with surgery. Nutr Hosp. 2013; 28(Suppl. 2): 47-52.

[5] Laursen D, Christensen K, Christensen U, Frølich A. Assessment of short and long-term outcomes of diabetes patient education using the health education impact questionnaire (HeiQ). BMC research notes. 2017; (10): 213

[6] Cox J, Coupland C. Diabetes treatments and risk of amputation, blindness, severe kidney failure, hyperglycemia, and hypoglycemia: an open cohort study in primary care. BMJ. 2016; 352: 1450.

[7] Alotaibi A, Perry L, Gholizadeh L, Ganmi A. Incidence and prevalence rates of diabetes mellitus in Saudi Arabia: An overview. Journal of Epidemiology and global health. 2017; 7(4): 211-218.

[8] Ganz M, Wintfeld N, Li Q, Alas, V, Langer J, Hammer M. The association of body mass index with the risk of type 2 diabetes: a case-control study nested in an electronic health records system in the United States. Diabetology \& metabolic syndrome. 2014; (6): 50.

[9] Naeem Z. Burden of diabetes mellitus in Saudi Arabia. International journal of health sciences. 2015 ; 9 (3) V: V1.

[10] Najarkolaei F, Pakpour A, Saffari M, Hosseini MS, Hajizadeh F, Chen H, Yekaninejad M. Determinants of lifestyle in Iranian adults with prediabetes: Applying the theory of planned behavior. Arch Iran Med. 2017; 20(4): 198 204.

[11] Boles A, Kandimalla R, Reddy H. Dynamics of Diabetes and Obesity: Epidemiological Perspective. Biochimica et Biophysica acta. Molecular basic of disease. 2017; 1863(5): 1026-1036.

[12] Verhulst M, Loos B, Gerdes V, Teeuw W. Evaluating all potential oral complications of diabetes mellitus. Frontiers in Endocrinol. 2019; (10): 56.

[13] Goldberg I. Diabetic Dyslipidemia: Causes and Consequences. The journal of clinical Endocrinology \& metabolism. 2001; 86(3): 965-971.

[14] Eltobgi A. Libya has the highest prevalence of diabetes, Mellitus type 2 in North Africa, and the Arab World. Endocrine Abstracts. 2009; (19): 138.

[15] Parmar M. Obesity and Type 2 diabetes mellitus. Integrative obesity and diabetes. 2018; 4(4): 1-2.

[16] Fletcher B, Gulanick M, Lamendola C. Risk factors for type 2 diabetes mellitus. J cardiovascular nursing. 2002; 16(2): 17-23.

[17] Classification and diagnosis of diabetes. American diabetes association. Diabetes Care. 2015; 38 (Suppl. 1): 8-16.

[18] Chawla R, Madhu S, Makkar B, Ghosh S, Saboo B, Karla S. and On behalf of the RSSDI-ESI Consensus Group RSSDIESI clinical practice recommendations for the management of type 2 diabetes mellitus 2020. Indian journal of endocrinology and diabetes. 2020; 24(1): 1-122.

[19] Elhwuegi A, Daraz A, Langa A, Bashaga N. Cross-sectional pilot study about the health status of diabetic patients in the city of Misurata, Libya. African health sciences. 2012; 12(1).

[20] Romdhane H, Ali S, Aissi W, Traissac, Skhiri H, Bougatef S, Maire B, Delpeuch F, Achour N. Prevalence of diabetes Northern African countries: the case of Tunisia. BMC Public Health. 2014; 14: 86. 
[21] Ashur S, Shah S, Soad Bosseri S, Fah T, Shamsuddin K. Libyan. Glycaemic control status among type 2 diabetic patients and the role of their diabetes coping behaviors: a clinic-based study in Tripoli, Libya. Journal of Medicine. 2016.

[22] Noureddine H, Nakhoul N, Galal A, Soubra L and Saleh M. Level of A1C control and its predictors among Lebanese type 2 diabetic patients. Ther Adv Endocrinol Metab. 2014; 5(3): 43-52.

[23] El-Shareif H. Quality of care for type 2 diabetes mellitus in Tripoli Medical Center: a retrospective study of 628 patients. Clinical Diabetology. 2017; 6 (6): 204-210. 